\title{
乌ூ
}

\section{Glass Transitions in Quasi-Two-Dimensional Suspensions of Colloidal Ellipsoids}

\author{
Zhongyu Zheng (郑中玉), Feng Wang (王峰), and Yilong Han (韩一龙)* \\ Department of Physics, Hong Kong University of Science and Technology, Clear Water Bay, Hong Kong, China
}

(Received 28 February 2011; published 1 August 2011)

\begin{abstract}
We observed a two-step glass transition in monolayers of colloidal ellipsoids by video microscopy. The glass transition in the rotational degree of freedom was at a lower density than that in the translational degree of freedom. Between the two transitions, ellipsoids formed an orientational glass. Approaching the respective glass transitions, the rotational and translational fastest-moving particles in the supercooled liquid moved cooperatively and formed clusters with power-law size distributions. The mean cluster sizes diverge in power law as they approach the glass transitions. The clusters of translational and rotational fastest-moving ellipsoids formed mainly within pseudonematic domains and around the domain boundaries, respectively.
\end{abstract}

DOI: 10.1103/PhysRevLett.107.065702

PACS numbers: 64.70.pv, 82.70.Dd

Colloids are outstanding model systems for glass transition studies because the trajectories of individual particles are measurable by video microscopy [1]. In the past two decades, significant experimental effort has been applied to studying colloidal glasses consisting of isotropic particles [1-5] but little to anisotropic particles [6]. The glass transition of anisotropic particles has been studied in three dimensions (3D) mainly through theory and simulation $[7,8]$. Molecular mode-coupling theory (MCT) predicts that particle anisotropy should lead to new phenomena in glass transitions $[9,10]$, and some of these have been observed in recent 3D simulations of hard ellipsoids [11,12]. Molecular MCT [10,13] suggests that hard ellipsoids with an aspect ratio $p>2.5$ in 3D can form an orientational glass in which rotational degrees of freedom become glass while the center-of-mass motion remains ergodic [9]. Such a "liquid glass" [14], in analogy to a liquid crystal, has not yet been explored in 3D or even 2D experiments. Anisotropic particles should also enable exploration of the dynamic heterogeneity in the rotational degrees of freedom. In the framework of MCT, glass transitions were studied in 2D systems of monodisperse hard disks, binary hard disks, and binary dipole particles [15], but the glass transitions of monodispersed particles have not yet been studied in 2D experiment and simulation. It is well known that monodispersed spheres can be quenched to a glass in 3D but hardly in $2 \mathrm{D}$ even at the fastest accessible quenching rate. Hence bidispersed or highly polydispersed spheres have been used in experiments [5,16,17], simulations [18], and theory [15] for 2D glasses. In contrast, we found that monodispersed ellipsoids of intermediate aspect ratio are excellent glass formers in 2D because their shape can effectively frustrate crystallization and nematic order.

Here we investigate the glass transition in monolayers of colloidal ellipsoids by using video microscopy. We measured the translational and rotational relaxation times, the non-Gaussian parameter of the distribution of displacements, and the clusters of cooperative fastestmoving particles. These results consistently showed that the glass transitions of rotational and translational motions occur in two different area fractions, defining an intermediate orientational glass phase.

The ellipsoids were synthesized by stretching polymethyl methacrylate spheres $[19,20]$. They had a small polydispersity of $5.6 \%$ with the semilong axis $a=$ $3.33 \mu \mathrm{m}$ and the semishort axes $b=c=0.56 \mu \mathrm{m}$. $3 \mathrm{mM}$ sodium dodecyl sulfate was added to stabilize ellipsoids, and the $>3 \mathrm{mM}$ ionic strength in the aqueous suspension made ellipsoids moderately hard particles with the Debye length $\sim 20 \mathrm{~nm}$. A monolayer of ellipsoids was strongly confined between two glass walls [20]. Light interference measurements showed that the wall separation varied by only $\sim 30 \mathrm{~nm}$ per $1 \mathrm{~mm}$ [20], so the walls could be considered as parallel within the field of view. The area fraction $\phi \equiv \pi a b \rho$, where $\rho$ is the number density averaged over all video frames. Twelve densities were measured in the range $0.20 \leq \phi \leq 0.81$. During the $3-6 \mathrm{~h}$ of measurements at each $\phi$, no drift flow or density change was observed. The thermal motion of the ellipsoids was recorded by using a charge-coupled device camera resolving $1392 \times 1040$ pixels at 1 frame per second for the highest five concentrations and at 3 frames per second for lower concentrations. The center-of-mass positions and orientations of individual ellipsoids were tracked by using our image processing algorithm [21]. The angular resolution was $1^{\circ}$, and the spatial resolutions were 0.12 and $0.04 \mu \mathrm{m}$ along the long and the short axes, respectively. More experimental details are in the Supplemental Materials [22].

At high densities the ellipsoids spontaneously formed small pseudonematic domains with branchlike structures each involving about $10^{2}$ particles; see Fig. S1 of the Supplemental Materials. The translational relaxation was characterized by the self-intermediate scattering function $F_{s}(q, t) \equiv\left\langle\sum_{j=1}^{N} e^{i \mathbf{q} \cdot\left[\mathbf{x}_{j}(t)-\mathbf{x}_{j}(0)\right]}\right\rangle / N$, where $\mathbf{x}_{j}(t)$ is the position of ellipsoid $j$ at time $t, N$ is the total number of 
particles, $\mathbf{q}$ is the scattering vector, and \langle\rangle denotes a time average. In Fig. 1(a), we chose $q_{m}=2.3 \mu \mathrm{m}^{-1}$ measured from the first peak position in the structure factor at high density. The rotational relaxation can be characterized by the $n$th order of the orientational correlation function $L_{n}(t) \equiv\left\langle\sum_{j=1}^{N} \cos n\left[\theta_{j}(t)-\theta_{j}(0)\right]\right\rangle / N$, where $n$ is a positive integer and $\theta_{j}$ is the orientation of ellipsoid $j . L_{n}(t)$ decays faster for larger $n$, and different choices of $n$ can yield the same glass transition point. $n=4$ in Fig. 1(b) was chosen so that $L_{n}(t)$ can be better displayed within our measured time scales. At high $\phi$, both $F_{s}\left(q_{m}, t\right)$ and $L_{4}(t)$ develop two-step relaxations, the characteristics upon approaching the glass transition. The short-time $\beta$ relaxation corresponds to motion within cages of neighboring particles, and the long-time $\alpha$ relaxation reflects structural rearrangement involving a series of cage breakings. According to MCT, the $\alpha$ relaxation follows $e^{-(t / \tau)^{\beta}}$ [15]. Figure 1(c) shows that $\beta$ decreases with density, indicating dynamic slowing-down upon supercooling [11,23].

MCT predicts that the relaxation time $\tau(\phi)$ diverges algebraically approaching the critical point $\phi_{c}: \tau(\phi) \sim$ $\left(\phi_{c}-\phi\right)^{-\gamma}$, where $\gamma=1 /(2 a)+1 /(2 b)$ [24]. Here $a$ and $b$ are the exponents in the critical-decay law [15] $F_{s}(q, t)=f_{q}^{c}+h_{q} t^{-a}$ and the von Schweidler law [15] $F_{s}(q, t)=f_{q}^{c}-h_{q}^{\prime} t^{b}$ at the initial stage of the $\beta$ relaxation and the crossover time to the $\alpha$ relaxation, respectively. The fitted $a_{T}=0.3 \pm 0.02$ and $b_{T}=0.63 \pm 0.02$ for $F_{s}\left(q_{m}, t\right)$ and $a_{\theta}=0.32 \pm 0.02$ and $b_{\theta}=0.65 \pm 0.02$ for $L_{4}(t)$ yield $\gamma_{T}=2.45 \pm 0.05$ and $\gamma_{\theta}=2.33 \pm 0.05$ for the translational and orientational correlations, respectively. These values are close to the $\gamma_{T}=2.3$ measured
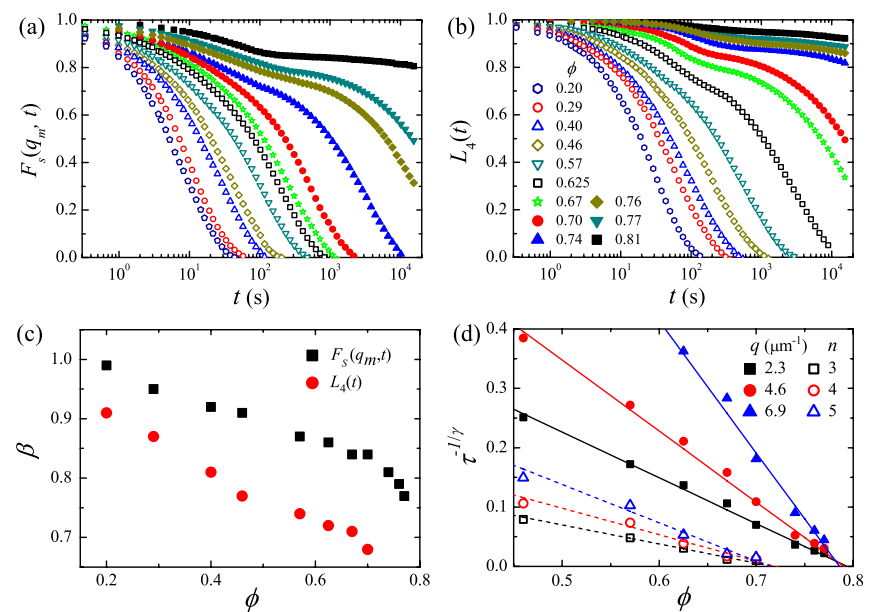

FIG. 1 (color online). (a) The self-intermediate scattering function $F_{s}(q, t)$ at $q_{m}=2.3 \mu \mathrm{m}^{-1}$ and (b) the orientational correlation $L_{4}(t)$ for different area fractions. (c) The exponent $\beta$ of the fitting function $e^{-(t / \tau)^{\beta}}$ for the long-time $F_{s}\left(q_{m}, t\right)$ and $L_{4}(t)$. (d) The fitted relaxation time $\tau(\phi) \sim\left(\phi_{c}-\phi\right)^{-\gamma}$. Solid symbols: Different choices of $q$ in $F(q, t)$ for the translational motion. Open symbols: Different choices of $n$ in $L_{n}(t)$ for the orientational motion. for 3D ellipsoids [12]. In Fig. 1(d), $\tau^{-1 / \gamma}$ is linear in $\phi$ for different choices of $q$ and $n$. Interestingly, all the scalings show that the glass transitions are at $\phi_{c}^{\theta}=0.72 \pm 0.01$ for rotational motion and $\phi_{c}^{T}=0.79 \pm 0.01$ for translational motion. This indicates three distinct phases: liquid $(\phi<$ 0.72 ), an intermediate orientational glass which is liquidlike in its translational degrees of freedom but glassy in its rotational degrees of freedom $(0.72<\phi<0.79)$, and the glass state for both degrees of freedom $(\phi>0.79)$.

Besides the extrapolations in Fig. 1(d), the existence of the orientational glass phase was verified from the nonGaussian parameters $\alpha_{2}(t)=\left\langle\Delta x^{4}\right\rangle /\left(3\left\langle\Delta x^{2}\right\rangle^{2}\right)-1$ of particle displacements $\Delta x$ during time $t$ [1]. In supercooled liquids, the distribution of $\Delta x$ is Gaussian at short and long times because the motions are diffusive, but it becomes non-Gaussian with long tails at the intermediate times due to cooperative out-of-cage displacements $[1,4,25]$. This behavior is reflected in the peak of $\alpha_{2}(t)$; see Fig. 2. As $\phi$ increases, the peak rises and shifts towards a longer time, indicating growing dynamic heterogeneity on approaching the glass transitions. In contrast, the glass phase lacks cooperative out-of-cage motions, so $\alpha_{2}(t)$ exhibits no distinct peak and declines with time [1]. Such a sharp change has been regarded as a characteristic of a glass transition in $3 \mathrm{D}$ [1]. Figure 2 clearly shows the glass transitions at $\phi_{c}^{\theta}=$ $0.72 \pm 0.02$ for rotational motion and at $\phi_{c}^{T}=0.79 \pm 0.02$ for translational motion. In Fig. 2(a), $\alpha_{2}^{\|}(t)$ is always greater than the corresponding $\alpha_{2}^{\perp}(t)$, indicating that the translational relaxations and cooperative out-of-cage motions are mainly along the long axes of the ellipsoids.

The two glass transitions can be further confirmed from the spatial distribution of the fastest-moving particles which characterizes the structural relaxation and dynamic heterogeneity [1]. In Fig. 3, the fastest-moving $8 \%$ of the particles are labeled in colors because the non-Gaussian long tail of the distribution of $\Delta x\left(t^{*}\right)$ covers about $8 \%$ of the population. Here $t^{*}$ corresponds to the maximum of $\alpha_{2}$ [1]. Different choices of $t$ and the percentage yield similar results. Neighboring fastest-moving ellipsoids form
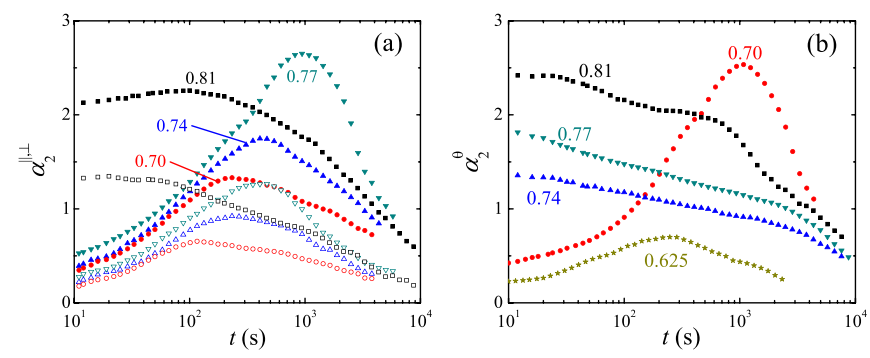

FIG. 2 (color online). (a) The non-Gaussian parameters of translational displacements along the long axis $\left[\alpha_{2}^{\|}(t)\right.$, solid symbols] and the short axis $\left[\alpha_{2}^{\perp}(t)\right.$, open symbols]. $\phi=$ $0.70,0.74,0.77,0.81$ as labeled in the figures. (b) The nonGaussian parameters of rotational displacements. 

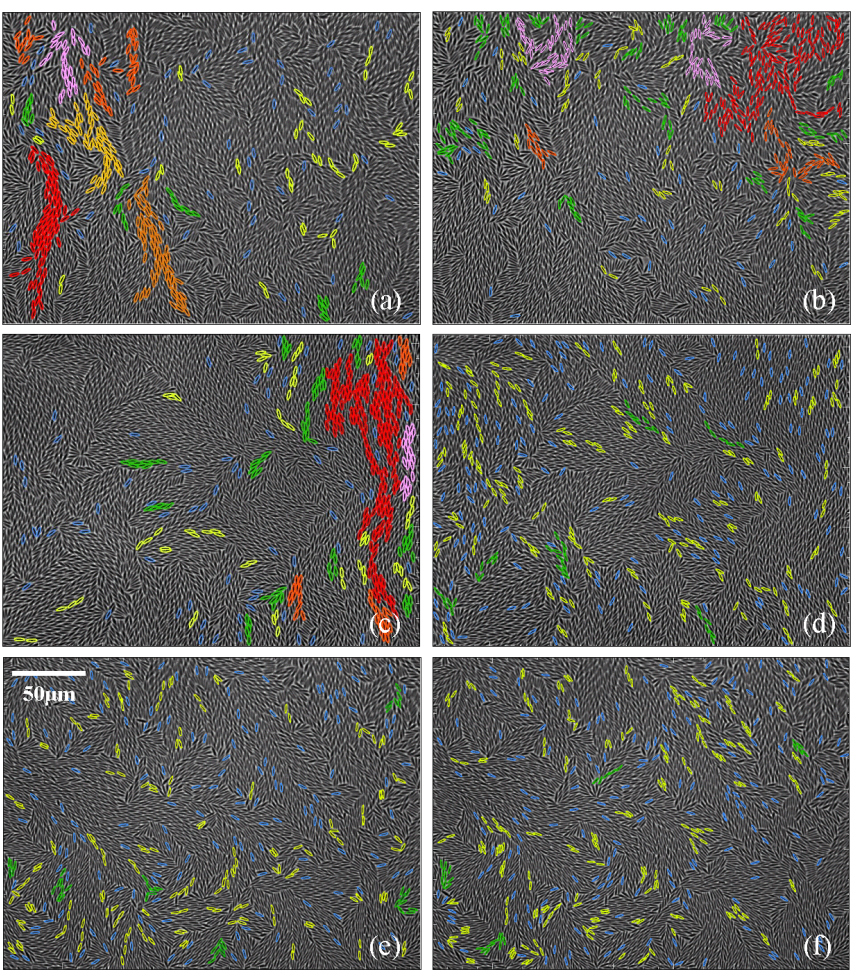

FIG. 3 (color online). The spatial distributions of the fastestmoving $8 \%$ of the particles (labeled in colors) in translational (a),(c),(e) and rotational (b),(d),(f) motions. Ellipsoids in the same cluster have the same color. (a),(b) The same frame at $\phi=$ 0.70 (supercooled liquid); (c),(d) the same frame at $\phi=0.77$ (orientational glass); (e),(f) the same frame at $\phi=0.81$ (glass) with $\sim 5500$ particles.

clusters and are labeled by using the same color. Here two ellipsoids are defined as neighbors if they overlap after being expanded by 1.5 times and their closest distance does not intersect a third particle. In the supersaturated liquid, most fast particles were strongly spatially correlated and formed large extended clusters; see Fig. 3 and Fig. S3 in the Supplemental Materials. This demonstrates that the $\alpha$ relaxation occurs by cooperative particle motion in both the translational and rotational degrees of freedom: When one particle moves, another particle moves closely following the first. The colloidal glasses, in contrast, show no discernible $\alpha$ relaxation, and the fastest particles in $\beta$ relaxation are randomly dispersed without forming large clusters [1], as observed in the 3D glass transition of colloidal spheres [1]. Figure 3 clearly depicts three regimes: Both the translational and rotational fast particles are distributed heterogeneously with large clusters at $\phi<0.72$; the rotational fast particles are dispersed homogeneously while the translational fast particles form large clusters at $0.72<\phi<0.79$; and both types of fast particles are dispersed homogeneously at $\phi>0.79$.

The spatial distributions of translational and rotational fast-particle clusters were anticorrelated. Figures 3(a) and 3(c) show that most translational fast
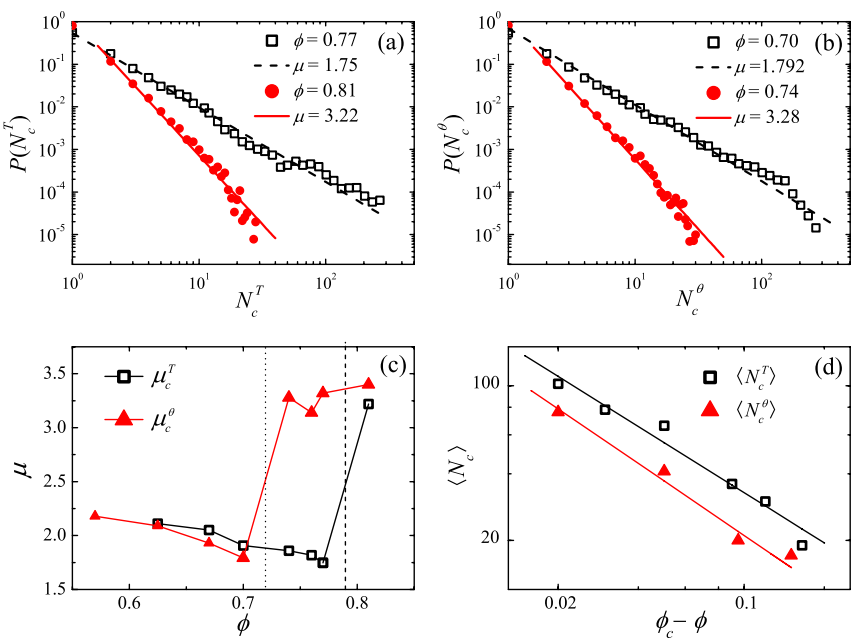

FIG. 4 (color online). The probability distribution functions for the cluster size of (a) translational and (b) rotational fastestmoving particles. The lines are the best fits of $P\left(N_{c}\right) \sim N_{c}^{-\mu}$. (c) The fitted exponents $\mu^{\theta}$ for rotational motions and $\mu^{T}$ for translational motions. The vertical dotted and dashed lines represent the glass transitions for rotational and translational motions, respectively. (d) The weighted mean cluster size $\left\langle N_{c}\right\rangle \sim$ $\left(\phi_{c}-\phi\right)^{-\eta}$, where $\phi_{c}^{\theta}=0.71$ and $\phi_{c}^{T}=0.79$.

particles belonged to a few large ribbonlike clusters aligned with their long axes within the pseudonematic domains. In contrast, the clusters of rotational fast particles formed branchlike structures extending over several small domains around the domain boundaries; see Fig. 3(b). Fast rotational ellipsoids moved between domains by cooperative rotational motion. This demonstrates that the nematic order within a domain facilitates translational relaxation while the orientational disorder near domain boundaries promotes rotational relaxation. Fast translational particles are responsible for the out-of-cage diffusion, while fast rotational particles are responsible for domain transformations such as splitting, merging, and rotating. All the phases in Figs. 3(a)-3(f) contain some isolated fast translational and rotational particles; they are mainly distributed at the domain boundaries with random orientations.

The cluster sizes of the fast particles, $N_{c}$, exhibit a power-law distribution $P\left(N_{c}\right) \sim N_{c}^{-\mu}$ as shown in Figs. 4(a) and 4(b). The fitted exponents $\mu$ for translational and rotational motions change dramatically near their respective glass transitions; see Fig. 4(c). The $\mu^{\theta, T}=2.0 \pm$ 0.2 for supersaturated liquids is close to the $\mu^{T}=2.2 \pm$ 0.2 estimated for hard spheres [1] and the $\mu^{T}=1.9 \pm 0.1$ for Lennard-Jones particles in 3D [25], while the $\mu^{\theta, T}=$ $3.2 \pm 0.1$ for glasses is close to the $\mu^{T}=3.1$ estimated for hard spheres in 3D [1]. Hence, $\mu \simeq 2.5$ might characterize such glass transitions in general. Figure 4(d) shows the weighted mean cluster size $\left\langle N_{c}\right\rangle=\sum N_{c}^{2} P\left(N_{c}\right)$ / $\sum N_{c} P\left(N_{c}\right)$ [1,25] at different densities. Both $\left\langle N_{c}^{\theta}\right\rangle$ and $\left\langle N_{c}^{T}\right\rangle$ diverge on approaching the corresponding 
$\phi_{c}:\left\langle N_{c}\right\rangle \sim\left(\phi_{c}-\phi\right)^{-\eta}$ with fitted $\eta^{\theta}=0.81$ and $\eta^{T}=$ 0.75 , indicating growing cooperative regions of mobile particles. Similar scaling and $\eta^{T}$ have been observed in a Lennard-Jones system [25], but the mechanism is not clear.

We did not observe nematic phase or semetic domains found in 3D spherocylinders [26] because (i) the elliptical shape facilitates particles changing orientation and forming branchlike structures at high densities [27], (ii) the $5.6 \%$ polydispersity promotes glass formation, and (iii) long-wavelength fluctuations are stronger in 2D than in 3D, which can more easily break the long-range order as described by the Mermin-Wagner theorem [28]. Ellipsoids with $p \sim 6$ appeared to be good glass formers, which can easily preempt any isotropic-nematic phase transition [29]. In contrast, the glass transition can be preempted by crystallization for $p \simeq 1$ in $2 \mathrm{D}$ or by an isotropic-nematic transition for rods with $p \gtrsim 25$ in 3D [8].

All of the measurements consistently showed that the glass transitions for ellipsoids with $p=6$ confined between two walls are at $\phi_{c}^{\theta}=0.72$ for rotational motion and at $\phi_{c}^{T}=0.79$ for translational motion. For longer ellipsoids with $p=9(a=5.9 \mu \mathrm{m}, b=c=0.65 \mu \mathrm{m})$, $\phi_{c}^{\theta}=0.60 \pm 0.02$ and $\phi_{c}^{T}=0.72 \pm 0.02$ were observed in the two-wall confinement. This suggests that the intermediate regime between $\phi_{c}^{\theta}$ and $\phi_{c}^{T}$ increases with the aspect ratio, which could be the reason why such an intermediate regime has not been observed in previous 3D simulations of ellipsoids with small aspect ratios $[12,30]$.

We conclude that colloidal ellipsoids in a quasi-2D system exhibit two glass transitions with an intermediate orientational glass. This behavior has been predicted in 3D by molecular MCT but not studied in 2D before. The two glass transitions in the rotational and translational degrees of freedom correspond to interdomain freezing and innerdomain freezing, respectively. The orientational glass regime appears to increase with the aspect ratio. Approaching the glass transitions, the structural relaxation time and the mean cluster size for cooperative motion diverge-typical features of a glass transition [2,24,25]. Interestingly, the translational and orientational cooperative motions are anticorrelated in space, which has not been predicted in theory or simulation. A similar two-step glass transition has been observed in a 3D liquid-crystal system and explained as the freezing of the orientations of the pseudonematic domains and the freezing of the translational motion within domains [31]. Here we directly observed the conjectured pseudonematic domains in Ref. [31]. These results at single-particle resolution shed new light on the formation of molecular glasses, especially at low dimensionality.

We thank Ning $\mathrm{Xu}$ and Penger Tong for the helpful discussion. This work was supported by HKUST Grant No. RPC07/08.SC04 and by GRF Grant No. 601208. *yilong@ust.hk

[1] E. R. Weeks, J. C. Crocker, A. C. Levitt, A. Schofield, and D. A. Weitz, Science 287, 627 (2000).

[2] W. Götze and L. Sjögren, Phys. Rev. A 43, 5442 (1991).

[3] W. van Megen and S. M. Underwood, Phys. Rev. Lett. 70, 2766 (1993).

[4] W. Kegel et al., Science 287, 290 (2000).

[5] Z. Zhang et al., Nature (London) 459, 230 (2009).

[6] P. J. Yunker et al., Phys. Rev. E 83, 011403 (2011).

[7] F. Stillinger and J. Hodgdon, Phys. Rev. E 50, 2064 (1994); V. Ilyin, E. Lerner, T.-S. Lo, and I. Procaccia, Phys. Rev. Lett. 99, 135702 (2007).

[8] G. Yatsenko and K. Schweizer, Langmuir 24, 7474 (2008).

[9] M. Letz, R. Schilling, and A. Latz, Phys. Rev. E 62, 5173 (2000).

[10] R. Schilling and T. Scheidsteger, Phys. Rev. E 56, 2932 (1997).

[11] C. De Michele, R. Schilling, and F. Sciortino, Phys. Rev. Lett. 98, 265702 (2007).

[12] P. Pfleiderer, K. Milinkovic, and T. Schilling, Europhys. Lett. 84, 16003 (2008).

[13] T. Franosch, M. Fuchs, W. Götze, M. R. Mayr, and A.P. Singh, Phys. Rev. E 56, 5659 (1997).

[14] R. Schilling, J. Phys. Condens. Matter 12, 6311 (2000).

[15] M. Bayer et al., Phys. Rev. E 76, 011508 (2007); D. Hajnal, J. Brader, and R. Schilling, Phys. Rev. E 80, 021503 (2009); D. Hajnal, M. Oettel, and R. Schilling, J. Non-Cryst. Solids 357, 302 (2011).

[16] H. König, R. Hund, K. Zahn, and G. Maret, Eur. Phys. J. E 18, 287 (2005).

[17] P. Yunker, Z. Zhang, K. B. Aptowicz, and A. G. Yodh, Phys. Rev. Lett. 103, 115701 (2009); S. Mazoyer, F. Ebert, G. Maret, and P. Keim, Europhys. Lett. 88, 66004 (2009).

[18] R. Speedy, J. Chem. Phys. 110, 4559 (1999).

[19] C. Ho, A. Keller, J. Odell, and R. Ottewill, Colloid Polym. Sci. 271, 469 (1993).

[20] Y. Han, A. Alsayed, M. Nobili, and A. G. Yodh, Phys. Rev. E 80, 011403 (2009).

[21] Z. Zheng and Y. Han, J. Chem. Phys. 133, 124509 (2010).

[22] See Supplemental Material at http://link.aps.org/ supplemental/10.1103/PhysRevLett.107.065702 for experimental details.

[23] T. Kawasaki, T. Araki, and H. Tanaka, Phys. Rev. Lett. 99, 215701 (2007).

[24] W. Götze and L. Sjögren, Rep. Prog. Phys. 55, 241 (1992); S. P. Das, Rev. Mod. Phys. 76, 785 (2004).

[25] C. Donati, S. C. Glotzer, P. H. Poole, W. Kob, and S. J. Plimpton, Phys. Rev. E 60, 3107 (1999).

[26] R. Ni, S. Belli, R. van Roij, and M. Dijkstra, Phys. Rev. Lett. 105, 088302 (2010).

[27] V. Narayan, N. Menon, and S. Ramaswamy, J. Stat. Mech. (2006) P01005.

[28] N.D. Mermin and H. Wagner, Phys. Rev. Lett. 17, 1133 (1966).

[29] J. A. Cuesta and D. Frenkel, Phys. Rev. A 42, 2126 (1990).

[30] S.-H. Chong, A.J. Moreno, F. Sciortino, and W. Kob, Phys. Rev. Lett. 94, 215701 (2005).

[31] H. Cang, J. Li, V. Novikov, and M. Fayer, J. Chem. Phys. 119, 10421 (2003) 